\title{
s-Degenerate Helices in 5-Dimensional Minkowski Space
}

\section{Zafer Şanlı}

\begin{abstract}
In this paper, it is characterized the 2-degenerate helices and 3-degenerate helices (that is, 2 and 3-degenerate curves with constant Cartan curvatures) in 5-dimensional Minkowski space and obtained that a classification of them.
\end{abstract}

Index Terms-Cartan curvatures, Cartan references, helices, s-degenerate curves.

\section{INTRODUCTION}

The geometry of null hypersurfaces in spacetimes has played an important role in the development general relativity, as well as in mathematics and physics of gravitation.

General null hypersurfaces, consist of investigating the curves that live in those hypersurfaces. In this sense, the null curves in Loretzian space forms has been studied by several authors( see [3],[4],[5])

However, in a null hypersurface there are many other curves distinct from the null ones. They are spacelike curves with a null higher derivative, that is, s-degenerate curves. These curves has been studied by A. Ferrandez, G. Gimenez, and P. Lucas(see [2]).

In this paper, it is characterized the 2-degenerate helices and 3-degenerate helices (that is, 2 and 3-degenerate curves with constant Cartan curvatures) in 5-dimensional Minkowski space and obtained that a classification of them.

\section{PRelaminaries}

Let $\mathrm{E}$ be a real vector space with a symmetric bilinear mapping $g: E \times E \rightarrow \mathbb{R}$. We say that $g$ is degenerate on $E$ if there exists a vector $\xi \neq 0$ in $E$ such that

$$
g(\xi, v)=0, \quad \text { for all } v \in E
$$

otherwise, $g$ is said to be non-degenerate. The radical (also called null space) of $E$, with respect to $g$, is the subspace $\operatorname{Rad}(E)$ of $E$ defined by

$\operatorname{Rad}(E)=\left\{\xi \in E: g(\xi, v)=0, v \in E_{;}\right\}$.

The dimension of $\operatorname{Rad}(E)$ is called the nullity degree of $g$ (or $E$ ) and is denoted by $r_{E}$.

If $F$ is a subspace of $E$, then we can consider $g_{E}$ the symmetric bilinear mapping on $F \times F$ obtained by restricting $g$ and define $F_{F}$ as the nullity degree of $F$ $\left(\right.$ or $\left.g_{s}\right)$. For simplicity, we will use 6 instead of $g$ or $g_{F}$.

A vector $W$ is said to be timelike, lightlike or spacelike provided that $g(w, v)<0_{x} \quad g(w, v)=0$ (and $\left.v \neq 0\right)$.

Zafer Şanlı, Department of Mathematics, Burdur Mehmet Akif Ersoy University, Burdur, Turkey or $g\left(\mathbb{W}_{w} \mathbb{W}\right)>0$, respectively. The vector $\mathbb{W}=0$ is assumed to be spacelike. A unit vector is a vector $u$ such that $g\left(u_{v} w\right)= \pm 1$.

Two vectors $u$ and $\mathbb{w}$ are said to be orthogonal, written

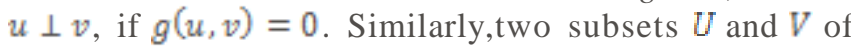
$\mathbb{E}$ are said to be orthogonal if $u \mathbb{N}$ for any $u \in \mathbb{U}$ and W $\in$ V.

Given two orthogonal subspaces $\mathbb{F}_{1}$ and $F_{2}$ in $\mathbb{E}$ with $F_{1} \cap F_{2}=\{0\}$, the orthogonal direct sum of $F_{1}$ and $F_{2}$ will be denoted by $\mathbb{F}_{1} \perp \mathbb{F}_{2}$.

Lemma 1 Let $(E, G)$ be a bilinear space and let $F$ be a hyperplane of $\mathbb{E}$. Let $F_{F}=\operatorname{dimRad}(F)$ and $r_{E}=\operatorname{dimRad}(E) \times$ Then the following statements hold:

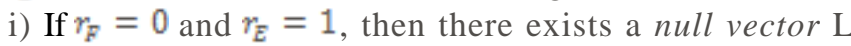
such that

$$
E=F \mathbb{L} \text { span }\{\mathbb{L}\} .
$$

ii) If $\widetilde{r}_{\mathbb{F}}=r_{\mathbb{E}}$ and $\widetilde{r}_{\mathbb{E}}=\left\{0_{0} 1\right\}$, then there exists a non-null unit vector $\mathbb{V}$ such that

$$
E=F \perp \operatorname{span}\{V\}
$$

Moreover, if $\operatorname{Rad}(E)=\{0\}$ then $V$ is unique, up to the sign.

iii) If $\tilde{P}_{\mathscr{E}}=\mathbb{1}$ and $\tilde{r}_{\mathbb{E}}=0$, and $F=F_{1} \perp \mathbb{L}$, where $\mathbb{L} \in \operatorname{Rad}(F)$ and $\mathbb{F}_{1}$ is non-degenerate, then there exists a unique null vector $N$ such that $\left\langle L_{s} N\right)=\varepsilon_{s} \varepsilon= \pm 1$, and

$$
E=\operatorname{span}\{\mathrm{L}\} \oplus \operatorname{span}(\mathrm{N}) \perp F_{1}
$$

\section{S-DEGENERATE CURVES}

Let $\left(M_{1}^{n}, \nabla\right)$ be an oriented Lorentzian manifold and let $\gamma: I \rightarrow M_{1}^{n}$ be a differentiable curve in $M_{1}^{n}$. For any vector field $\mathrm{V}$ along $\gamma_{s}$ let $\mathrm{V}$ ' be the covariant derivation of $\mathrm{V}$ along $\gamma$. Write $E_{i}(t)=\operatorname{span}\left\{\gamma^{\prime}(t), \gamma^{\prime \prime}(t), \ldots, \gamma^{(i)}(t)\right\}, \quad$ where $t \in I$ and $i=1,2, \ldots, n$. Let $d$ be the number defined by $d=\max \left\{i: \operatorname{dim}\left(E_{i}(t)\right)=i\right.$ for all $\left.t\right\}$.

Definition 2 With the above notations, the curve $\gamma: I \rightarrow M_{1}^{n}$ is said to be an s-degenerate (or s-lightlike) curve if for all $1 \leq i \leq d, \operatorname{dim}\left(\operatorname{Rad}\left(E_{i}(t)\right)\right.$ is constant for all $t$, and there exists $s, 0<s<d$, such that $\operatorname{Rad}\left(E_{s}\right) \neq\{0\}$ and $\operatorname{Rad}\left(E_{j}\right)=\{0\}$ for all $j<s$.

Theorem 3 Let $\gamma: I \rightarrow \mathcal{M}_{1}^{n}, n=m+2$, be an s-degenerate unit curve, $s>1$, and suppose that 
$\left\{\gamma^{\prime}(t), \gamma^{n}(t), \ldots, \gamma^{n}(t)\right\}$ spans $T_{\gamma(t)} \mathcal{M}_{1}^{n}$, for all $t$. Then there exists a unique Frenet frame satisfying equations: $\gamma^{\prime}=W_{1}$

$W_{1}^{\prime}=k_{1} W_{2}$

$W_{i}^{\prime}=-k_{i-1} W_{i-1}+k_{i} W_{i+2}, 2 \leq i \leq s-2$

$W_{s-1}^{\prime}=-k_{s-2} W_{s-2}+L$

$L^{\prime}=k_{s-1} W_{s}$

$W_{s}^{\prime}=\varepsilon k_{s} L-\varepsilon k_{s-1} N$

$N^{\prime}=-\varepsilon W_{s-1}-k_{s} W_{s}+k_{s+1} W_{s+1}$

$W_{s+1}^{\prime}=-\varepsilon k_{s+1} L+k_{s+2} W_{s+2}$

$W_{j}^{\prime}=-k_{j} W_{j-1}+k_{j+1} W_{j+1}, s+2 \leq j \leq m-1$

$W_{m}^{\prime}=-k_{m} W_{m-1}$

for certain functions $\left\{k_{1}, k_{2}, \ldots, k_{m}\right\}$.

Definition 4 An $s$-degenerate curve, $s>1$, satisfying the above conditions is said to be an s-degenerate Cartan curve. The reference and curvature functions given by Theorem 3 will be called the Cartan reference and Cartan curvatures of $\gamma$, respectively.

Observe that when $m>s$ then $\varepsilon=-1$ and $k_{i}>0$ for $i \neq s_{y}$ and $k_{m}>0\left(k_{m}<0\right.$,resp. $)$ according to $\left\{\gamma^{\prime}, \gamma^{n}, \ldots, \gamma^{(n)}\right\}$ is positively or negatively oriented, respectively. However, when $m=s$ then $\varepsilon=-1$ or $\varepsilon=1$ according to

$\left\{\gamma^{\prime}, \gamma^{n}, \ldots, \gamma^{(n)}\right\}$

is positively or negatively oriented , respectively, and $k_{i}>0$ for $i \neq s$.

Definition 5 An s-degenate helix in $M_{1}^{n}$ is an s-degenerate Cartan curve having constant Cartan curvatures.

Theorem 6 Let $\gamma: I \rightarrow \mathbb{R}_{1}^{n}$ be an s-degenerate Cartan curve and $D_{t}$ denote the covariant derivative in $\mathbb{R}_{1}^{n}$ along $\gamma$. Then for any vector field $V$ along $\gamma$ we have $D_{t} V=V^{\prime}$, where $\langle$,$\rangle denotes the standart metric in \mathbb{R}_{1}^{n}$. If $\left\{W_{1}, \ldots, W_{s-1}, L, W_{s}, N, W_{s+1}, \ldots, W_{m}\right\}$ is the Cartan reference, then equations write down as follows:

$\gamma^{\prime}=W_{1}$,

$W_{1}^{\prime}=k_{1} W_{2}$,

$W_{i}^{\prime}=-k_{i-1} W_{i-1}+k_{i} W_{i+2}, 2 \leq i \leq s-2$,

$W_{s-1}^{\prime}=-k_{s-2} W_{s-2}+L$,

$L^{\prime}=k_{s-1} W_{s}$,

$W_{s}^{\prime}=\varepsilon k_{s} 1-\varepsilon k_{s-1} N$,

$N^{\prime}=-\varepsilon W_{s-1}-k_{s} W_{s}+k_{s+1} W_{s+1}$,

$W_{s+1}^{\prime}=-\varepsilon k_{s+1} L+k_{s+2} W_{s+2}$,

$W_{j}^{\prime}=-k_{j} W_{j-1}+k_{j+1} W_{j+1}, s+2 \leq j \leq m-1$,

$W_{m}^{\prime}=-k_{m} W_{m-1}$.

IV. S-DEGENERATE HELICES IN $\mathrm{R}_{1}{ }^{5}$

Proposition 7 Let $\gamma: I \rightarrow \mathbb{R}_{1}^{5}$ be an 2-degenerate Cartan curve. If $y$ is a helix, $\gamma$ satisfies following differential equation:

$$
\gamma^{(6)}=2 \varepsilon \sigma_{1} \sigma_{2} \gamma^{(4)}+\varepsilon \sigma_{1} \sigma_{a}^{2} \gamma^{m}+\sigma_{1}^{2} \gamma^{n}
$$

Proof: By Theorem 6, we have

$\gamma^{s}=W_{1}$

$W_{1}^{\prime}=L$

$L^{s}=\sigma_{1} W_{2}$

$W_{2}^{\prime}=\varepsilon \sigma_{2} L-\varepsilon \sigma_{1} N$

$N^{s}=-\varepsilon W_{1}-\sigma_{2} W_{2}+\sigma_{3} W_{3}$

$W_{\mathrm{a}}^{\prime}=-\sigma_{\mathrm{a}} W_{2}$.

Cartan curvatures of $y$ are constant, since $y$ is a helix curve. By differentiating both sides of the Eq. (1), and using Eq.(2) and Eq. (3), we have

$$
\gamma^{x s}=L
$$

and

$$
\gamma^{n s s}=\sigma_{1} W_{2} .
$$

By taking derivatives of this equation, and using Eq. (4), Eq. (5) and Eq. (6) we obtain the following differential equation

$$
\gamma^{[(6)}=2 \varepsilon \sigma_{1} \sigma_{2} \gamma^{[4]}+\varepsilon \sigma_{1} \sigma_{a}^{2} \gamma^{m}+\sigma_{1}^{2} \gamma^{m}
$$

Proposition 8 Let $\gamma: I \rightarrow \mathbb{R}_{1}^{5}$ be an 3-degenerate Cartan curve. If $y$ is a helix, $y$ satisfies following differential equation:

$\gamma^{(6)}-\left(2 \varepsilon \sigma_{2} \sigma_{a}-\sigma_{1}^{2}\right) \gamma^{[4]}-\left(2 \varepsilon \sigma_{1}^{2} \sigma_{2} \sigma_{a}+\sigma_{2}^{2}\right) \gamma^{m}=0$

Proof: By Theorem 6, we have

$\gamma^{s}=W_{1}$

$W_{1}^{p}=L$

$L^{\prime}=\sigma_{1} W_{2}$

$W_{2}^{\prime}=\varepsilon \sigma_{2} L-\varepsilon \sigma_{1} N$

$N^{s}=-\varepsilon W_{1}-\sigma_{2} W_{2}+\sigma_{3} W_{3}$

$W_{a}^{\prime}=-\sigma_{a} W_{2}$.

Then, by a straightforward computations, the result is obtained as in the the proof of the Proposition 7.

\section{REFERENCES}

[1] Z. Şanl1, "On Degenerate Helices ," , M.Sc. Thesis, Süleyman Demirel University, Graduate School of Applied and Natural Sciences, Department of Mathematics, 2009

[2] A. Ferrandez,A. Gimenez, P. Lucas, "s-Degenerate Curves in Lorentzian Space Forms", Journal of Geometry and Physics, 40, 116-129, 2003.

[3] K.L. Duggal, A., Bejancu., Lightlike Submanifolds of semi-Riemanian Manifolds and Applications, Kluwer Academic Publishers, The Netherlands, 1996

[4] W.B. Bonnor "Null curves in Minkowski Spacetime" Tensor N.S., 20, 229-242, 1969.

[5] A. Ferrandez, A. Gimenez, P. Lucas, "Null helices in Lorentzian space forms", Int. Journal of Modern Physics, 2002.

[6] B. O’Neill, Semi-Riemann Geometry with Applications to Relativity, Academic Press, New York 